\title{
Thermorésistance anormale de spores bactériennes chauffées par injection directe dans la vapeur
}

\author{
par \\ O. CERF et J. HERMIER \\ Institut National de la Recherche Agronomique, Station Centrale de Recherches \\ Laitières et de Technologie des Produits Animaux \\ 78350 Jouy-en-Josas (France)
}

\begin{abstract}
A b stract
Abnormal heat resistance of bacterial spores heated by direct injection into steam
\end{abstract}

Spores of Clostridium tyrobutyricum, Bacillus cereus and B. stearothermophilus were heated in a pilot-plant U.H.T. sterilizer, of the milk-into-steam type. Temperatures were chosen between 102 and $115^{\circ} \mathrm{C}$, and the mean holding time was about $10 \mathrm{sec}$. Working fluids were buffer, whole milk or skim milk. Distribution function for residence times was determined by means of methylene blue as a tracer. Theoretical sporicidal effectiveness was calculated for each strain and each temperature, using heat resistance values previously determined by heating spores in T.D.T. tubes. With Cl. tyrobutyricum and $B$. cereus the experimental sporicidal effectiveness was always higher, from 1.2 to 7 times, than the computed effectiveness. Furthermore, with $\mathrm{Cl}$. tyrobutyricum spores, the T.D.T. curve is modified : $\mathrm{z}$ increases (13० $\mathrm{C}$ instead of $9.8^{\circ} \mathrm{C}$ in T.D.T. tubes.) B. stearothermophilus spores heated in the same U.H.T. sterilizer were partially inactivated at a temperature at which heating in ampoules results in an activation.

\section{Rés u m é}

Des spores de Clostridium tyrobutyricum, Bacillus cereus et $B$. stearothermophilus ont été chauffées par injection dans la vapeur au moyen d'un stérilisateur semi-industriel de type U.H.T. Les températures choisies étaient comprises entre 102 et $115^{\circ} \mathrm{C}$ pour un temps de séjour moyen de $10 \mathrm{~s}$ environ. Les liquides de chauffage étaient une solution tampon ou du lait entier ou écrémé. La répartition des temps de séjour a été déterminée avec, comme traceur, du bleu de méthylène. L'efficacité stérilisatrice théorique a été calculée pour chaque souche 
et chaque température en utilisant les valeurs des paramètres de thermorésistance mesurés par chauffages en ampoules. Pour $\mathrm{Cl}$. tyrobutyricum et $B$. cereus l'efficacité stérilisatrice expérimentale s'est révélée être supérieure, de 1,2 à 7 fois, à l'efficacité stérilisatrice théorique. De plus, dans le cas de Cl. tyrobutyricum, ceci s'accompagne d'une modification de la courbe de temps de destruction thermique, caractérisée par une augmentation de la valeur $\mathrm{z}$, de $9,8^{\circ} \mathrm{C}$ pour les chauffages en ampoules à $13^{\circ} \mathrm{C}$ pour les chauffages en continu avec injection dans la vapeur. Les spores de B. stearothermophilus elles-mêmes sont partiellement inactivées, à une température ou au contraire, les chauffages en ampoules provoquent une activation.

\section{INTRODUCTION}

Dans un premier travail (Cerf, Bergère et Hermier, 1967), nous avions déterminé les caractéristiques de thermorésistance des spores de $\mathrm{Cl}$. tyrobutyricum au moyen de chauffages en ampoules scellées à des températures comprises entre 80 et $105^{\circ} \mathrm{C}$. Dans le présent travail nous étendons cette étude au cas de chauffages à des températures supérieures à $100^{\circ} \mathrm{C}$ dans un appareillage de type U.H.T. (ultra-haute température) fonctionnant par injection directe. L'effet de ce mode de traitement a jusqu'à présent été étudié sur les spores de Bacillus subtilis (Hermier, Vergé et Grosclaude, 1959 ; Edwards, Busta et Speck, 1965), de B. stearothermophilus (Busta, 1967 ; Franklin, Underwood, Perkin et Burton, 1970) et de Cl. sporogenes PA 3679 (Busta, 1967) qui présentent une thermorésistance très supérieure à celle des spores de Cl. tyrobutyricum.

\section{MATERIEL ET METHODES}

\section{1) Souches}

Cl. tyrohutyricum CNRZ 510 et 514, dont les caractéristiques ont été préalablement publiées (Cerf et al., 1967).

B. stearothermophilus FS 1518 (ATCC 7953), B. cereus 111 isolée au laboratoire à partir de lait.

\section{2) Production des spores}

Les spores de $\mathrm{Cl}$. tyrobutyricum ont été produites comme décrit précédemment (Cerf et al., 1967) sur milieu TGE en boyau de dialyse à $37^{\circ} \mathrm{C}$. Celles de $B$. stearothermophilus ont été produites sur milieu de Wang (1963) dans des boîtes de Roux à $55^{\circ} \mathrm{C}$. Les spores de $B$. cereus ont été produites sur le milieu de Hermier (1962) dans des boîtes de Roux à $30^{\circ} \mathrm{C}$. Pour les trois espèces, les spores sont 
lavées par cinq centrifugations successives dans de l'eau distillée stérile, puis conservées sous forme de suspension concentrée à $10^{10}$ spores par $\mathrm{ml}$ dans de l'eau distillée stérile à $4^{\circ} \mathrm{C}$.

\section{3) Numération des spores}

Les spores de $\mathrm{Cl}$. tyrobutyricum sont dénombrées par la méthode du nombre le plus probable avec 10 tubes par dilution, sur milieu de Hirsch et Grinsted (1954) recouvert de $1 \mathrm{~cm}$ de vaspar. Les tubes sont incubés à $37^{\circ} \mathrm{C}$ pendant un mois. Les spores des trois souches de Bacillus sont dénombrées sur milieu de Wang (1963) réparti en boîtes de Petri, à raison de trois boîtes par dilution. Le milieu de Wang est préparé avec $15 \mathrm{~g} / \mathrm{l}$ de gélose seulement. Les boîtes sont incubées à $30^{\circ} \mathrm{C}$ pendant $40 \mathrm{~h}$ pour $B$. cereus, à $55^{\circ} \mathrm{C}$ pendant $40 \mathrm{~h}$ pour B. stearothermophilus.

\section{4) Chauffage des spores}

\section{a) Chauffage en ampoules}

Les courbes de survie et les courbes de temps de destruction thermique (TDT) ont été établies en suivant la technique d'Esty et Meyer (1922), par chauffage de $1,5 \mathrm{ml}$ de suspension de spores en solution tampon phosphate de potassium $0,02 \mathrm{M}$ à $\mathrm{pH}=7,0$ dans des ampoules en verre ordinaire $(100 \mathrm{~mm}$ de long, $8 \mathrm{~mm}$ de diamètre extérieur scellées à la flamme, et chauffées dans un bain de glycérine thermostaté à $\pm 0,02^{\circ} \mathrm{C}$ (Haake Ultrathermostat modèle NBS). Après chauffage et refroidissement dans de l'eau à la température de la glace fondante, les ampoules sont ouvertes aseptiquement, et les spores de $1 \mathrm{ml}$ de leur contenu sont dénombrées. Au moins 3 ampoules sont examinées pour chaque chauffage.

\section{b) Chauffage par injection dans la vapeur}

L'appareil utilisé est une installation pilote dérivée du stérilisateur Laguilharre (Hermier et al., 1959). La figure 1 présente le schéma de principe de cet appareil, tel qu'il a été utilisé pour ces essais. Le liquide de travail est pris dans le réservoir A et mis sous pression par la pompe centrifuge $\mathrm{B}$ à la sortie de laquelle est situé un débitmètre (précision du débit : \pm 3 p. 100). Il passe dans le préchauffeur tubulaire $C$, avant de pénétrer par une buse dans la section de chauffage $D$. La buse injecte le liquide dans de la vapeur, en le dispersant sous forme de fines gouttelettes dont une partie ruisselle le long des parois du caisson $\mathrm{D}$, et dont l'autre partie tombe directement au fond de celui-ci. Dans la section de chauffage, la température du traitement est déterminée à $\pm 0,5^{\circ} \mathrm{C}$ près par de la vapeur saturante sous pression. Cette température est maintenue jusqu'au robinet $\mathrm{E}$. A partir de E, la température est commandée par réglage du vide partiel qui règne dans le refroidisseur F. Pour ces essais, les températures en $\mathrm{C}$ et en $\mathrm{F}$ ont été maintenues voisines, de sorte que le débit 


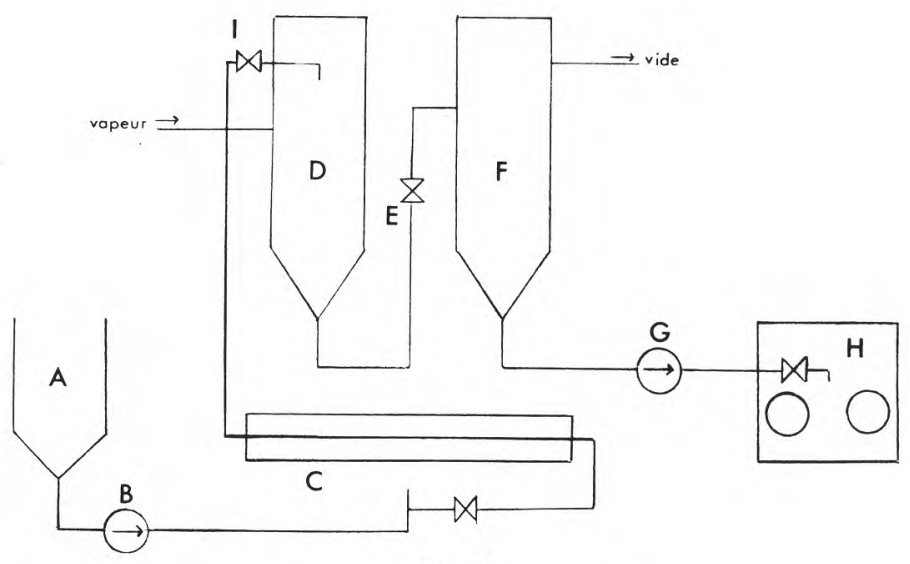

fig. 1

Stérilisateur U.H.T. utilisé pour les essais. Voir dans le texte la signification des symboles.
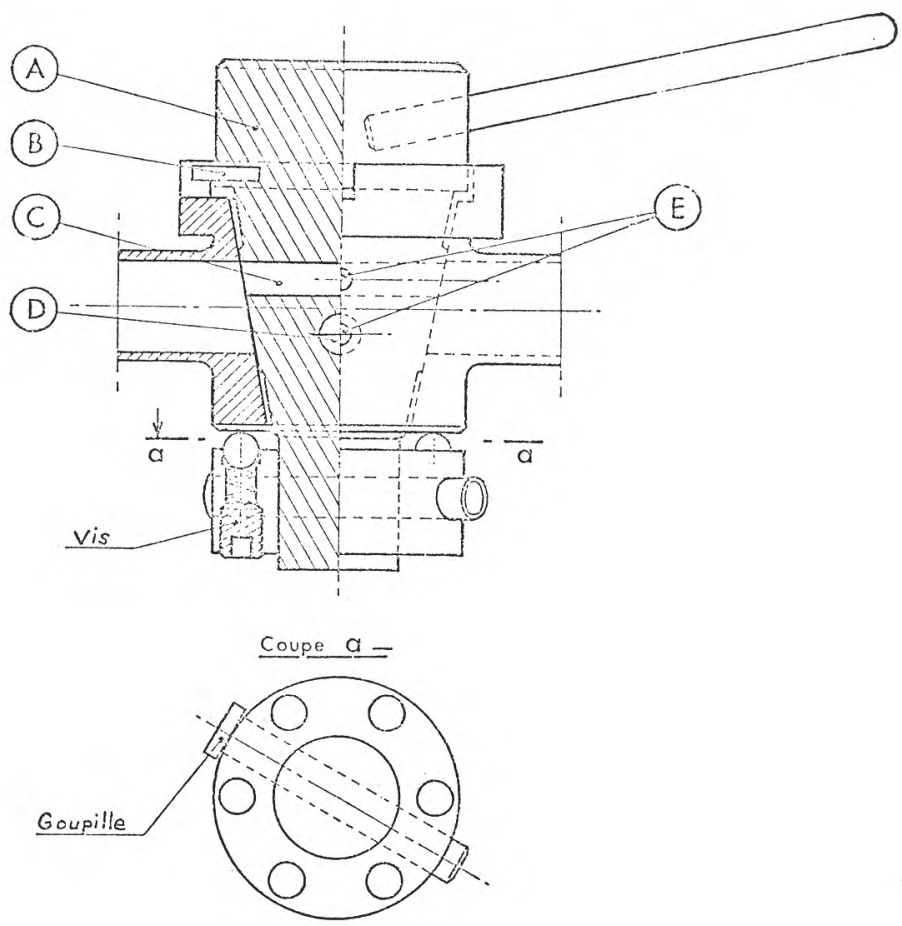

fig. 2

Vanne utilisée pour l'injection du marqueur et des spores dans le stérilisateur.

Voir dans le texte la signification des symboles. 
en $\mathrm{C}$ et en $\mathrm{F}$ était le même à 0,1 p. 100 près. La pompe centrifuge aseptique $\mathrm{G}$ dirige le liquide refroidi au point de prélèvement $\mathrm{H}$ situé dans une boîte à gants aseptique.

Les spores ont été introduites dans cet appareil d'une manière discontinue au moyen d'une vanne située à l'entrée de la section de chauffage, en I sur la figure 1. Cette vanne (fig. 2) possède un boisseau $\mathrm{A}$ en poly-tétra-fluoro-éthylène percé de deux lumières $\mathrm{C}$ et $\mathrm{D}$. Les axes de ces lumières sont perpendiculaires, et le boisseau est disposé de manière à ce que l'une des lumières laisse passer le liquide de travail (lumière $\mathrm{C}$ sur la figure 2 ). L'autre lumière (D sur la figure 2) a alors son axe vertical. Le corps de la vanne est percé de deux orifices $\mathrm{E}$ de manière à ce que l'on puisse accéder à celle des lumières qui est verticale, ici la lumière D. Il est donc possible de vider cette dernière du liquide qu'elle contient au moyen d'une seringue, et d'y introduire avec une autre seringue $1 \mathrm{ml}$ de la solution de marqueur ou d'une suspension de spores. Une butée B est fixée sur le boisseau. La forme du corps de la vanne permet à ce dernier une rotation d'un quart de tour. L'injection est simplement réalisée en faisant tourner la vanne d'un quart de tour. Le moment et la durée des injections étaient mesurés grâce à des contacts placés dans la butée B. Les impulsions électriques étaient enregistrées par le " topeur " d'un millivoltmètre Sefram enregistreur (modèle GRVAT). La durée de rotation du boisseau est en moyenne de $0,18 \mathrm{~s}$ $(0,1$ à $0,3 \mathrm{~s})$.

Au cours de l'ensemble des essais, le débit de l'appareil était réglé à $1,9 \mathrm{1} / \mathrm{mn}$ et les températures en $\mathrm{D}$ et $\mathrm{F}$ étaient de $65^{\circ} \mathrm{C}$.

c) Milieux de chauffage

Il a été utilisé une solution tampon phosphate de potassium $0,02 \mathrm{M}$ à $\mathrm{pH} \mathrm{7,0,} \mathrm{du} \mathrm{lait} \mathrm{écrémé} \mathrm{et} \mathrm{du} \mathrm{lait} \mathrm{à} 34 \mathrm{~g} / 1$ de matière grasse, de $\mathrm{pH}$ 6,6. Après passage dans l'appareil, le $\mathrm{pH}$ de la solution tampon remontait d'environ 0,3 unité $\mathrm{pH}$.

\section{5) Mesure des temps de séjour}

La répartition des temps de séjour a été mesurée à l'aide d'un traceur, le bleu de méthylène en solution aqueuse à 0,1 p. 100 injectée en I de la même façon que les spores. L'élution du colorant a été suivie au moyen d'une cellule photo-émissive à gaz ayant son maximum de sensibilité à $800 \mathrm{~nm}$. (Automation industries modèle Ce C 3). La cellule était placée sous le robinet $\mathrm{E}$ ou à l'entrée de la boîte à gants H. Le courant fourni par la cellule était enregistré sur le millivoltmètre.

\section{6) Calcul de l'efficacité stérilisatrice}

Connaissant la valeur du taux de destruction des spores $(\mathrm{k})$ à une température donnée, et la courbe de répartition de leurs temps de 
séjour $(H(t)$, t étant le temps), il est possible de calculer l'efficacité stérilisatrice d'un traitement thermique. En effet le nombre total de spores traitées est $\int_{0}^{\infty} H(t) d t$. Le nombre de spores survivant au traitement est $\int_{0}^{\infty} 10-\mathrm{ktH}(\mathrm{t}) \mathrm{dt}$ (Burton, 1958). L'efficacité stérilisatrice (L) est le logarithme du rapport du nombre total injecté au nombre de survivants. Burton (1958) proposait une méthode de résolution graphique. Pour rendre les calculs plus précis et plus aisés, un program-

\section{TABLEAU 1}

Programme de calcul de l'efficacité stérilisatrice (Fortran IV).

(La présentation des données est précisée dans les commentaires du programme).

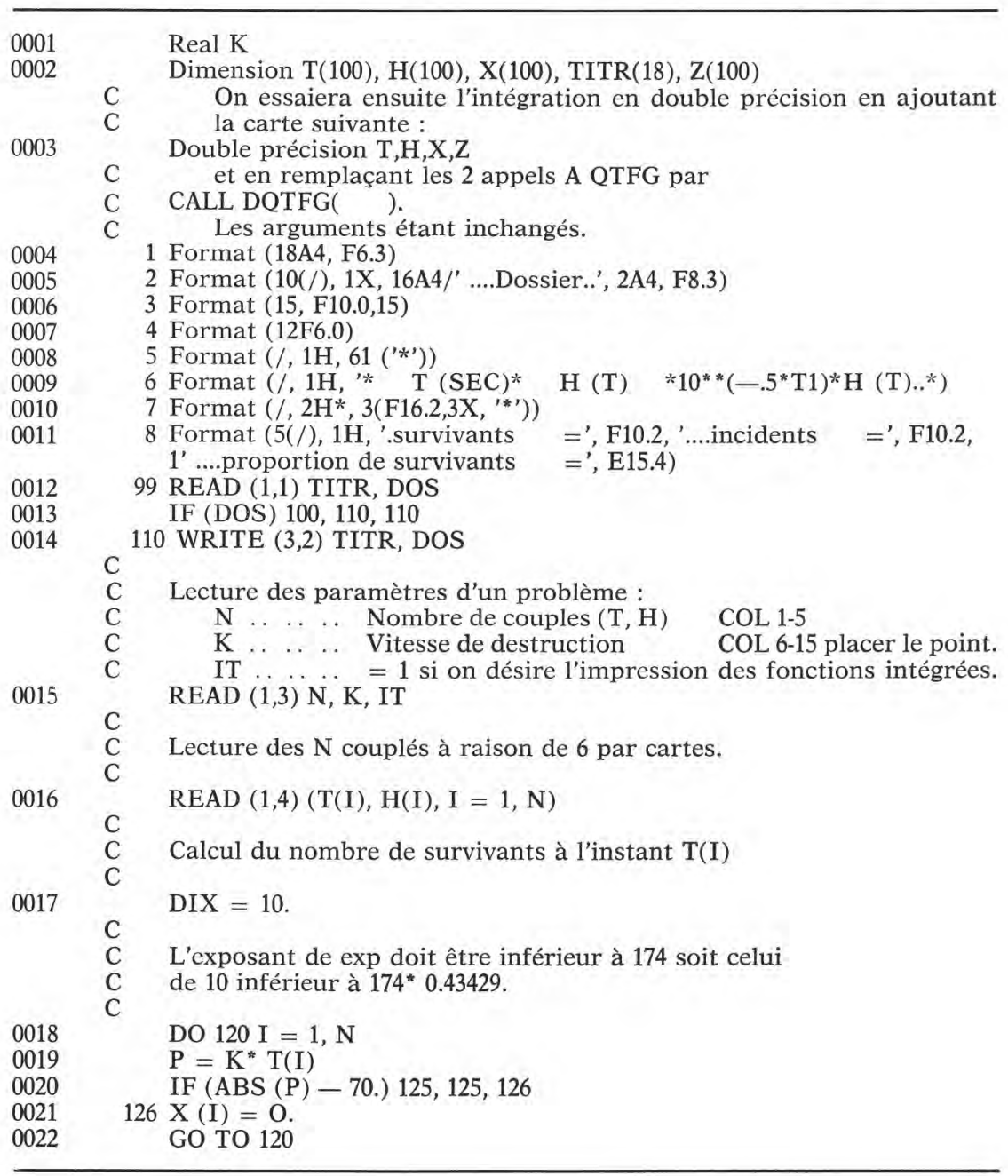




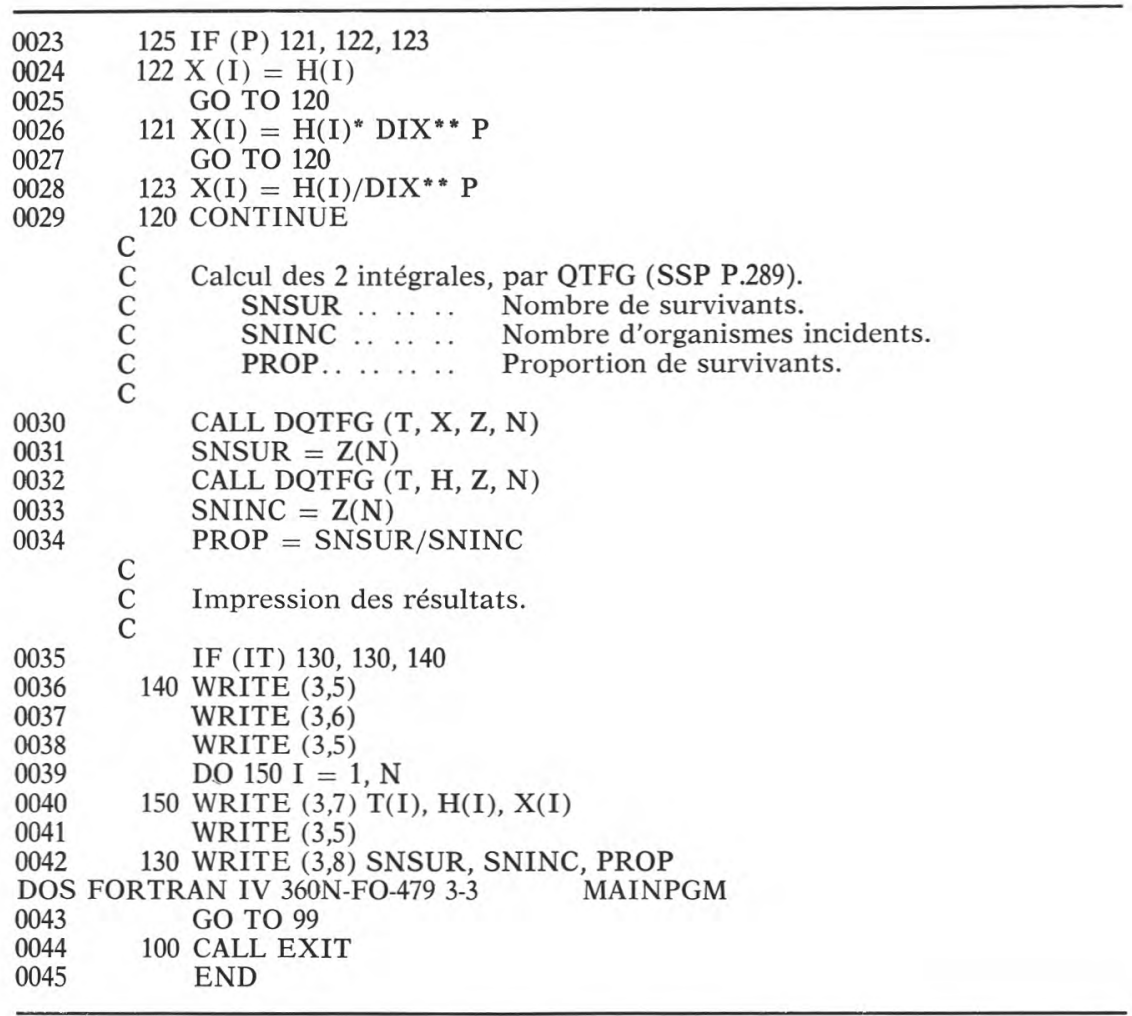

TABLEAU 2

Exemple de résultat fourni par le programme du tableau 1

\begin{tabular}{|c|c|c|}
\hline $\mathrm{T}(\mathrm{SEC})$ & $\mathrm{H}(\mathrm{T})$ & $10^{* *}\left(-.5^{*} \mathrm{~T}\right) * \mathrm{H}(\mathrm{T}) .$. \\
\hline 1.65 & 95.00 & 14.21 \\
\hline 1.75 & 210.00 & 28.00 \\
\hline 1.85 & 360.00 & 42.79 \\
\hline 1.95 & 510.00 & 54.02 \\
\hline 2.05 & 630.00 & 59.48 \\
\hline 2.15 & 694.00 & 58.39 \\
\hline 2.25 & 713.00 & 53.47 \\
\hline 2.35 & 720.00 & 48.12 \\
\hline 2.45 & 718.00 & 42.77 \\
\hline 2.55 & 700.00 & 37.16 \\
\hline 2.65 & 630.00 & 29.81 \\
\hline 2.75 & 530.00 & 22.35 \\
\hline 2.95 & 325.00 & 10.89 \\
\hline 3.15 & 210.00 & 5.59 \\
\hline 3.35 & 136.00 & 2.87 \\
\hline 3.75 & 60.00 & 0.80 \\
\hline Survivants & \multicolumn{2}{|l|}{$=53.78$} \\
\hline Incidents & \multicolumn{2}{|l|}{$=832.55$} \\
\hline Proportion de survivants & \multicolumn{2}{|l|}{$=0.6460 \mathrm{E}-01$} \\
\hline
\end{tabular}


me de calcul automatique a été mis au point par le Laboratoire de Biométrie du Centre National de Recherches Zootechniques (tab. 1). Dans l'exemple numérique donné par Burton (1958), cet auteur trouvait pour la proportion de survivants $6,33 \cdot 10^{-2}$ : notre méthode indique $6,46 \cdot 10^{-2}$ (tab. 2). Cette disparité est due à l'imprécision inhérente aux méthodes manuelles.

\section{RESULTATS}

\section{1) Thermorésistance des spores employées}

Les valeurs caractéristiques de la thermorésistance ( $\mathrm{D}$ et $\mathrm{z}$ ) déterminées par chauffages en ampoules sont données dans les tableaux 3 et 4 pour les souches utilisées. Les courbes de survie, établies sur

\section{TABLEAU 3}

Caractéristiques de thermorésistance des spores chauffées en ampoules scellées, en suspension dans une solution tampon phosphate $0,02 \mathrm{M}, \mathrm{pH} 7,0$

\begin{tabular}{|c|c|c|c|c|}
\hline \multirow{2}{*}{ Souche } & \multicolumn{2}{|c|}{$\mathrm{D}(\mathrm{mn}) \mathrm{à}$} & \multirow{2}{*}{$\begin{array}{l}\text { Intervalle des } \\
\text { températures } \\
\text { étudiées }\end{array}$} & \multirow{2}{*}{$\mathrm{z}\left({ }^{\circ} \mathrm{C}\right.$} \\
\hline & $90^{\circ} \mathrm{C}$ & $120^{\circ} \mathrm{C}$ & & \\
\hline Cl. tyrobutyricum 510 & 21 & & $\begin{array}{l}80-97 \\
85-105\end{array}$ & $\begin{array}{l}8,25 \\
9,8\end{array}$ \\
\hline B. cereus 111 & 44 & & $85-100$ & 9,8 \\
\hline B. stearothermophilus FS 1518 & & 10 & $80-130$ & 7,5 \\
\hline
\end{tabular}

\section{TABLEAU 4}

Caractéristiques de thermorésistance des spores chauffées en ampoules scellées, en suspension dans du lait écrémé à $\mathrm{pH}$ 6,6

\begin{tabular}{c|c|c|c}
\hline Souche & $\mathrm{D}_{90}(\mathrm{mn})$ & $\begin{array}{c}\text { Intervalle des } \\
\text { températures étudiées }\end{array}$ & $\mathrm{z}\left({ }^{\circ} \mathrm{C}\right)$ \\
\hline & & $80-97$ & 9,25 \\
Cl. tyrobutyricum 510 & 6,6 & $85-101$ & 9,9 \\
Cl. tyrobutyricum 514 & 14 & & \\
\hline
\end{tabular}


plus de 4 réductions décimales (logarithme du nombre de survivants en fonction du temps) et les courbes de temps de destruction thermique (TDT) (logarithme du temps de réduction décimale en fonction de la température) peuvent être assimilées à des droites (coefficient de sécurité 0,05 ).

\section{2) Détermination des temps de séjour dans la section de chauffage}

Avec l'appareillage de mesure monté sur le stérilisateur, le logarithme de la concentration d'une solution en eau distillée de bleu de méthylène est proportionnelle à la différence de potentiel dans l'intervalle de concentration $5 \cdot 10^{-2}$ à $3,9 \cdot 10^{-4} \mathrm{~g} / \mathrm{ml}$. D'autre part, après chauffage dans le stérilisateur à la température de $120^{\circ} \mathrm{C}$, la densité optique d'une solution de bleu de méthylène en eau distillée est en moyenne inférieure de 2 p. 100 seulement à celle de la solution non chauffée. Donc, dans les conditions expérimentales utilisées, après transformation des indications du voltmètre en concentration, le bleu de méthylène peut être employé comme traceur. Une courbe de répartition des temps de séjour à la température de $111^{\circ} \mathrm{C}$ est donnée à titre d'exemple sur la figure $3 \mathrm{~A}$. Elle représente la moyenne de trois essais. Le débit d'entrée et de sortie étant resté le même pour tous les essais, les différences que l'on observe entre les courbes obtenues à diverses températures proviennent du fait que la quantité

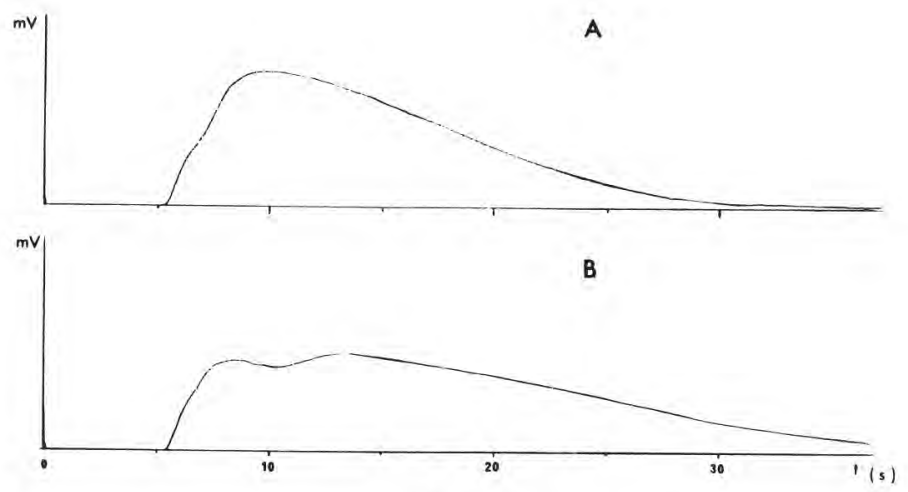

fig. 3

Exemples de courbes de répartition des temps de séjour dans la section de chauffage du stérilisateur. Le zéro de l'échelle des temps correspond à l'injection du marqueur.

A. circuit propre.

B. circuit entartré.

Ces courbes correspondent au réglage suivant du stérilisateur : température du préchauffage et du caisson sous vide : $65^{\circ} \mathrm{C}$, température de la section de chauffage $111^{\circ} \mathrm{C}$, débit $1,9 \mathrm{l} / \mathrm{mn}$. 
de vapeur condensée varie avec la température de l'essai. Cependant, le temps de séjour minimum varie peu et se situe à $5 \mathrm{~s}( \pm 0,25 \mathrm{~s})$.

Quand le liquide introduit dans l'appareil est de l'eau ordinaire et que la durée d'un essai se prolonge au-delà d'une demi-heure, l'aspect de la courbe de répartition des temps de séjour est considérablement modifié sans que le temps de séjour minimum le soit (fig. 3B), par suite de l'entartrage des parois de l'appareil. La première bosse du " dos de chameau " doit correspondre au passage direct des gouttelettes, la deuxième bosse aux goutelettes qui ruissellent de plus en plus lentement le long des parois. Rien de ceci n'apparaît lorsque le liquide de travail est de l'eau permutée ou distillée. Dans ce qui suit, les résultats mentionnés ont été obtenus avec de l'eau distillée ou permutée ; pour les essais avec du lait, leur durée a été limitée à une demi-heure.

\section{3) Choix du moment et de la durée de prélèvement à la sortie du stérilisateur}

La figure 4 montre la courbe de passage au point de prélèvement d'une solution de bleu de méthylène injectée à l'entrée de la section de chauffage, réglée à $111^{\circ} \mathrm{C}$ (courbe $\mathrm{A}$ ). La même courbe en moyenne a été obtenue pour des températures de traitement comprises entre 102 et $120^{\circ} \mathrm{C}$. Le passage du bleu de méthylène est comparé dans la même figure avec le passage d'une suspension de spores de B. stearothermophilus injectée dans les mêmes conditions. Pour ce dernier essai, la température de traitement était de $111^{\circ} \mathrm{C}$, température à laquelle toutes les spores restent viables lors de chauffages en ampou-

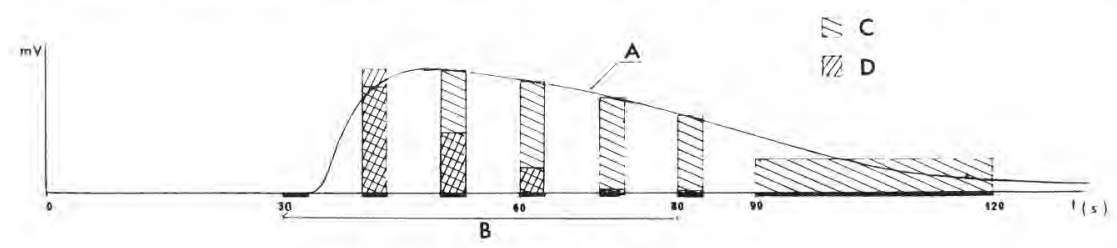

fig. 4

Passage du colorant et des spores de B. stearothermophilus au point de prélèvement. Le zéro de l'échelle des temps correspond à l'injection du colorant ou des spores.
A. courbe de passage du colorant.
B. période de prélèvement correspondant au passage de 70 p. 100 du colorant.
C. histogramme représentant le pourcentage de colorant passant au point de prélèvement pendant les périodes indiquées par un trait gras.
D. histogramme représentant le pourcentage de spores viables effectivement prélevées au cours d'un essai pendant les pé- riodes indiquées par un trait gras.

Les histogrammes $\mathrm{C}$ et $\mathrm{D}$ sont superposés. 
les. Les résultats sont portés sur la figure 4. Sur cette figure, l'aire totale sous la courbe A représente la quantité totale de colorant qui passe au point de prélèvement. Les rectangles $\mathrm{C}$ représentent la quantité de colorant qui passe au point de prélèvement pendant la période indiquée par un trait gras sur l'axe des abscisses (3 s environ, c'est-à-dire le temps nécessaire pour prélever $100 \mathrm{ml}$ ). L'aire de chacun de ces rectangles représente un certain pourcentage de l'aire totale sous la courbe A.

Sur la figure 4 , les rectangles $D$ représentent les pourcentages de spores qui ont été prélevés pendant la période indiquée par un trait gras, pourcentages calculés par rapport au nombre total de spores injectées. Si les spores transitent au point de prélèvement de la même façon que le colorant, les rectangles $\mathrm{C}$ et les rectangles $\mathrm{D}$ devront se superposer exactement. Or on voit sur la figure que la surface totale des rectangles $\mathrm{D}$ est inférieure à celle des rectangles C. Cela signifie que l'on n'a pu récolter autant de spores viables que l'on en avait injectées.

De même, lorsque l'on recueille la totalité du liquide dans la période 30 - $80 \mathrm{~s}$ (B) après l'injection du bleu de méthylène ou des spores de $B$. stearothermophilus, on constate que l'on recueille, d'une manière reproductible, 32 p. 100 de spores viables au lieu de 70 p. 100 du bleu de méthylène.

Cette discordance entre les deux méthodes de détermination des temps de séjour pouvait être attribuée soit à une inactivation partielle des spores de $B$. stearothermophilus, soit au fait que les spores effectuaient un parcours plus rapide ou plus lent que les molécules de bleu de méthylène. D'une part on n'a trouvé aucune spore dans le prélèvement effectué entre la $30^{\mathrm{e}}$ et $33^{\mathrm{e}} \mathrm{s}$, et on n'a trouvé que 2,5 p. 100 des spores injectées dans le liquide recueilli entre la $80^{\text {e }}$ et la $120^{\mathrm{e}} \mathrm{s}$ suivant l'injection. D'autre part, on a injecté un nombre suffisamment grand de spores de $B$. subtilis $\left(9.10^{\circ}\right)$ et de $C l$. tyrobutyricum $\left(10^{10}\right)$ pour que l'on puisse déterminer directement au microscope la concentration en spores de l'eau au point de prélèvement : les nombres récoltés entre la $30^{\mathrm{e}}$ et $1 \mathrm{la} 80^{\mathrm{e}} \mathrm{s}$ n'étaient pas significativement différents des nombres prévus, c'est-à-dire 70 p. 100 des spores injectées.

Ces essais montrent que le parcours effectué par les spores dans l'appareil est identique à celui du bleu de méthylène. Cette démonstration ne semble pas avoir été faite jusqu'à présent (Aiba, Humphrey et Millis, 1965). Donc, les spores de B. stearothermophilus sont partiellement inactivées par chauffage à $111^{\circ} \mathrm{C}$ dans le stérilisateur. $\mathrm{Ce}$ résultat était inattendu puisqu'il a été vérifié qu'un chauffage en ampoules à $111^{\circ} \mathrm{C}$ pendant $0,8 \mathrm{mn}$ en tampon phosphate $0,02 \mathrm{M}$ à pH 7,0 ne modifiait pas le nombre de spores viables. Pour les essais suivants, le moment du prélèvement a été fixé entre la $30^{\mathrm{e}}$ et la $80^{\mathrm{e}} \mathrm{s}$ après l'injection. La proportion de traceur ainsi récolté était dans ces conditions de $70 \pm 7$ p. 100 . 


\section{4) Thermorésistance des spores injectées dans la vapeur}

L'efficacité stérilisatrice L du stérilisateur vis-à-vis de $\mathrm{Cl}$. tyrobutyricum souche 514 a été déterminée expérimentalement pour des températures de chauffage comprises entre 102 et $115^{\circ} \mathrm{C}$. Dans le tableau 5 , les valeurs expérimentales de $\mathrm{L}$ sont comparées aux valeurs

TABLEAU 5

Efficacité stérilisatrice du traitement avec injection dans la vapeur sur les spores de $\mathrm{Cl}$. tyrobutyricum 514

\begin{tabular}{|c|c|c|c|c|}
\hline \multirow{2}{*}{ Milieu de chauffage } & \multirow{2}{*}{$\begin{array}{c}\text { Température } \\
\left({ }^{\circ} \mathrm{C}\right)\end{array}$} & \multicolumn{2}{|c|}{ Efficacité stérilisatrice } & \multirow{2}{*}{$\begin{array}{l}\mathrm{L}_{\mathrm{e}} \\
\mathrm{L}_{\mathrm{c}}\end{array}$} \\
\hline & & $\underset{\text { (expérimentale) }}{\mathrm{L}_{\mathrm{e}}}$ & $\begin{array}{c}\mathrm{L}_{\mathrm{c}} \\
\text { (calculée) }\end{array}$ & \\
\hline Tampon phosphate & $\begin{array}{l}102 \\
111 \\
115\end{array}$ & $\begin{array}{r}0,5 \\
2,9 \\
>7\end{array}$ & $\begin{array}{l}0,2 \\
1,6 \\
3,2\end{array}$ & $\begin{array}{r}2,5 \\
1,8 \\
>2,2\end{array}$ \\
\hline Lait entier & $\begin{array}{l}105 \\
111 \\
115\end{array}$ & $\begin{aligned} & 1,0 \\
& 4,6 \\
>> & 7\end{aligned}$ & $\begin{array}{l}0,8 \\
2,6 \\
5,2\end{array}$ & $\begin{array}{r}1,2 \\
1,8 \\
>1,3\end{array}$ \\
\hline Lait écrémé & $\begin{array}{l}102 \\
105 \\
111 \\
115\end{array}$ & $\begin{array}{r}1,0 \\
1,7 \\
3,3 \\
>7\end{array}$ & $\begin{array}{l}0,4 \\
0,8 \\
2,6 \\
5,2\end{array}$ & $\begin{array}{r}2,5 \\
2,1 \\
1,3 \\
>1,3\end{array}$ \\
\hline
\end{tabular}

TABLEAU 6

Efficacité stérilisatrice du traitement avec injection dans la vapeur sur les spores chauffées en tampon phosphate

\begin{tabular}{|c|c|c|c|c|}
\hline \multirow{2}{*}{ Souche } & \multirow{2}{*}{$\begin{array}{c}\text { Température } \\
\left({ }^{\circ} \mathrm{C}\right)\end{array}$} & \multicolumn{2}{|c|}{ Efficacité stérilisatrice } & \multirow{2}{*}{$\begin{array}{l}\mathrm{L}_{\mathrm{e}} \\
\mathrm{L}_{\mathrm{c}}\end{array}$} \\
\hline & & $\underset{\text { (expérimentale) }}{\mathrm{L}_{\mathrm{e}}}$ & $\begin{array}{c}\mathrm{L}_{\mathrm{c}} \\
\text { (calculée) }\end{array}$ & \\
\hline Cl. tyrobutyricum 510 & $\begin{array}{l}102 \\
105 \\
111\end{array}$ & $\begin{array}{r}4,2 \\
5,3 \\
>7\end{array}$ & $\begin{array}{l}1,2 \\
2,3 \\
7,5\end{array}$ & $\begin{array}{l}3,5 \\
2,3 \\
-\end{array}$ \\
\hline B. cereus 111 & $\begin{array}{l}102 \\
105 \\
111\end{array}$ & $\begin{array}{l}0,7 \\
0,7 \\
1,7\end{array}$ & $\begin{array}{l}0,1 \\
0,2 \\
0,8\end{array}$ & $\begin{array}{l}7 \\
3,5 \\
2,1\end{array}$ \\
\hline
\end{tabular}


de L calculées d'après les valeurs de $\mathrm{D}$ et $\mathrm{z}$ obtenues après chauffages en ampoules aux températures indiquées dans les tableaux 3 et 4 . Les valeurs expérimentales sont toujours supérieures aux valeurs calculées, quels que soient la température et le milieu de chauffage (tampon, lait entier ou écrémé). Sauf deux exceptions (chauffages à $105^{\circ} \mathrm{C}$ de lait entier et $111^{\circ} \mathrm{C}$ de lait écrémé), le rapport de la valeur expérimentale de $L$ à la valeur calculée est supérieur à 1,8 .

Pour vérifier que cette apparente diminution de la thermorésistance n'était pas la propriété d'une souche aberrante, l'efficacité stérilisatrice de l'appareillage a été déterminée vis-à-vis d'une autre souche de $\mathrm{Cl}$. tyrobutyricum (510) et d'une souche de $B$. cereus, espèce dont les spores sont en général peu thermorésistantes. Le chauffage a été effectué uniquement en tampon phosphate. Comme le montre le tableau 6, les valeurs expérimentales de L sont toujours supérieures aux valeurs calculées, quelles que soient la température et la souche utilisées. Le rapport entre valeur expérimentale et valeur calculée est soit du même ordre de grandeur que pour $\mathrm{Cl}$. tyrobutyricum souche 514 soit supérieure, en particulier à $102^{\circ} \mathrm{C}$. Il n'y a donc pas de relation entre la thermorésistance dans l'appareil à injection dans la vapeur et la thermorésistance déterminée en ampoules à des températures inférieures à $100^{\circ} \mathrm{C}$.

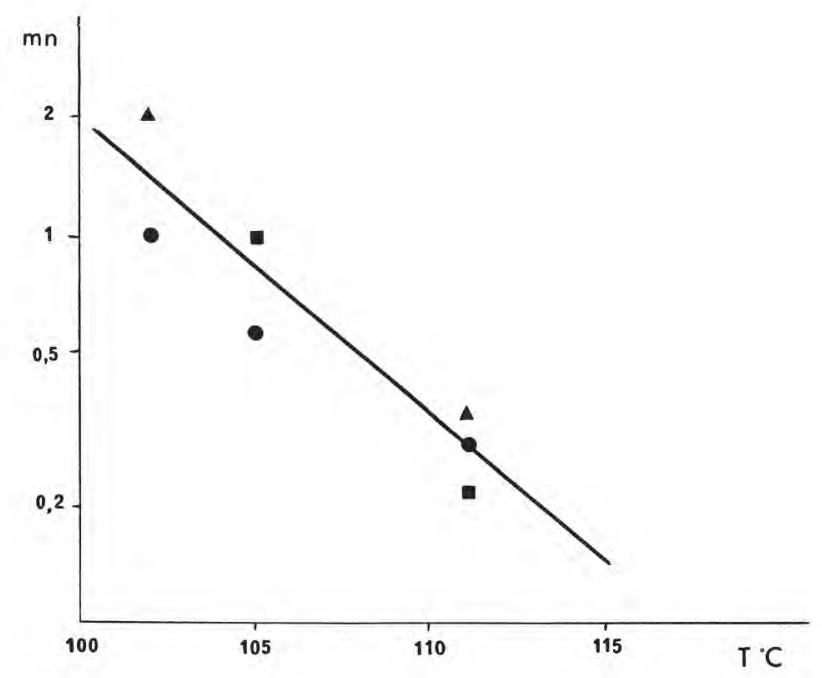

fig. 5

Courbe des temps de destruction thermique (TDT) des spores de Cl. tyrobutyricum 514 injectées dans la vapeur. Une durée de $1 \mathrm{mn}$ a été prise arbitrairement pour le temps de séjour moyen des spores dans la section de chauffage, dans le but de déterminer le coefficient de température, soit $\mathrm{z}=13^{\circ} \mathrm{C}$.

Les points correspondent à des chauffages effectués en solution tampon $(\boldsymbol{\Lambda})$, en lait entier ( $)$ ou en lait écrémé $(\bullet)$. 
Si l'on connaissait avec précision le temps de séjour moyen caractéristique de l'appareillage, il serait possible de connaître l'ordre de grandeur des temps de réduction décimale correspondant à chaque résultat. Mais la section de chauffage n'a pas une forme géométrique simple, on ignore le volume que le liquide y occupe et la proportion de vapeur qui est réellement en contact avec le liquide à stériliser. Cependant en prenant arbitrairement une valeur pour le temps de séjour moyen, on peut tracer une courbe de temps de destruction thermique (TDT), dont la position dépend de cette valeur, mais dont la pente, et donc le $\mathrm{z}$, en est indépendante. Ceci a été fait pour $\mathrm{Cl}$. tyrobutyricum 514 (fig. 5). La valeur de $\mathrm{z}$ obtenue est de $13^{\circ} \mathrm{C}$ au lieu des $9,8^{\circ} \mathrm{C}$ obtenus lors du chauffage en ampoules (tab. 3 ).

\section{DISCUSSION}

La résistance des spores de $C l$. tyrobutyricum au chauffage par injection dans la vapeur est très inférieure à celle prévue à partir des déterminations de thermorésistance de spores chauffées en ampoules. Le même phénomène a été retrouvé bien que beaucoup moins marqué, chez deux autres espèces, $B$. cereus et $B$. stearothermophilus. Plusieurs raisons peuvent être invoquées pour expliquer cette résistance anormalement faible. On a vérifié qu'elle n'était pas due à l'appareillage utilisé : spores anormalement retenues dans l'appareil, irrégularités dans le débit ou la température de chauffage, irrégularités aux prélèvements. D'ailleurs le fait que l'importance de l'abaissement de la thermorésistance est fonction de la souche exclut tout effet d'ordre purement mécanique.

En tenant compte des variations des temps de séjour minimum $( \pm 5$ p. 100$)$ des temps de prélèvements $( \pm 7$ p. 100$)$ et des variations de température (qui peuvent provoquer une variation de \pm 5 p. 100 de la thermorésistance), la précision relative des mesures serait de \pm 17 p. 100 aux températures non létales où l'on recueille 70 p. 100 $( \pm 7$ p. 100$)$ des spores injectées et elle serait de \pm 10 p. 100 aux températures inactivantes où l'on recueille à coup sûr 100 p. 100 des spores survivantes. Il conviendrait de tenir compte aussi de la variation de thermorésistance qui se produit lorsque le milieu de chauffage constitué par une solution tampon voit son $\mathrm{pH}$ modifié par l'injection de vapeur. Dans le cas de Cl. tyrobutyricum 514 il y a ainsi une diminution de thermorésistance de 10 p. 100 environ. Mais l'ensemble de ces causes d'imprécision technique ne suffit pas à rendre compte de l'ensemble du phénomène que nous avons décrit, qui se traduit par une diminution et jamais par une augmentation de la thermorésistance. Par ailleurs il a été vérifié que la vapeur n'apportait pas de facteurs inhibiteurs de la germination de la spore ni de la croissance végétative. 
En ce qui concerne les spores de $C l$. tyrobutyricum, nous avons observé non seulement une destruction plus forte que celle prévue par le calcul (souches 510 et 514), mais aussi un accroissement de la valeur de $\mathrm{z}$ (souche 514 ). Un résultat analogue a été trouvé par Busta (1967) avec des spores de $\mathrm{Cl}$. sporogenes $\left(\mathrm{z}=18-20^{\circ} \mathrm{C}\right.$ au lieu de $8-14^{\circ} \mathrm{C}, \mathrm{D}_{121}=7 \mathrm{~s}$ au lieu de $60 \mathrm{~s}$ ). On sait que l'utilisation de la notion de $\mathrm{z}$, coefficient de température de la courbe de temps de destruction thermique (TDT), n'est licite que dans un intervalle de température de $30^{\circ} \mathrm{C}$ environ. Certains auteurs préfèrent utiliser la notion d'énergie d'activation $\mathrm{E}$ d'Arrhénius. $\mathrm{E}$ et $\mathrm{z}$ sont liés par une équation simple (Pflug et Schmidt, 1968). Si nous calculons E à propos de $\mathrm{Cl}$. tyrobutyricum 514 dans l'intervalle de températures $85-105^{\circ} \mathrm{C}$ et que nous en déduisons $\mathrm{z}$ pour l'intervalle $105-120^{\circ} \mathrm{C}$, nous trouvons $10,6^{\circ} \mathrm{C}$, valeur bien inférieure à la valeur expérimentale. Cette dernière ne peut donc être expliquée totalement par la loi d'Arrhénius. Cette augmentation de la valeur de $\mathrm{z}$ avec la température pourrait être rapprochée de celle observée par Miller et Kandler (1967) et par Burton (1970) si nous ne savions que l'augmentation de la valeur de $\mathrm{z}$ est accompagnée ici par une diminution de la valeur de $\mathrm{D}$ aux températures où les mesures ont été faites à la fois en ampoules et par injection dans la vapeur.

Dans l'état actuel de nos connaissances, aucune explication satisfaisante ne peut être fournie pour ces modifications simultanées des valeurs de $\mathrm{D}$ et de $\mathrm{z}$. Peut-être pourrait-on avancer l'hypothèse suivante : en raison de la mise en équilibre quasi-instantanée à la température de chauffage, des constituants de la spore de conductivité thermique différente varieraient de volume indépendamment les uns des autres, entraînant une distorsion des structures sporales. Si effectivement la rapidité des échanges thermiques est la cause de cet effet destructeur, celui-ci devrait se retrouver à la suite de chauffages indirects avec échanges thermiques rapides. C'est bien ce qui se produit avec les spores de $C l$. tyrobutyricum souche 514 chauffées dans des tubes capillaires $\left(\mathrm{z}=11,8^{\circ} \mathrm{C}\right.$ au lieu de $9,8^{\circ} \mathrm{C}$, D $\mathrm{D}=4 \mathrm{mn}$ au lieu de $21 \mathrm{mn}$; Cerf, 1969). Cependant, en ce qui concerne le chauffage de spores ou de formes végétatives de bactéries dans des tubes capillaires, de très nombreux auteurs (par exemple Wilder et Nordan (1957) pour Cl. sporogenes PA 3679) avaient toujours constaté la similitude des résultats avec les chauffages en ampoules.

En ce qui concerne les spores de B. cereus, comme pour les deux souches de $\mathrm{Cl}$. tyrobutyricum, leur destruction par l'injection dans la vapeur est plus forte que prévue, et comme pour la souche 514 , leur $\mathrm{z}$ semblerait plus élevé ( $14^{\circ} \mathrm{C}$ au lieu de $9,8^{\circ} \mathrm{C}$ en ampoules). Toutefois ce résultat doit être considéré comme peu précis en raison du nombre limité de données.

En ce qui concerne les spores de B. stearothermophilus, nous avons observé qu'elles ont été inactivées par l'injection dans la vapeur par un couple temps-température auquel elles sont théorique- 
ment insensibles. Cela est à rapprocher tout d'abord des résultats de Busta (1967) qui observait avec la même souche, mais à des températures plus élevées, une thermorésistance moins forte lors de chauffages avec injection de vapeur $\left(D_{130}=14 \mathrm{~s}\right.$ au lieu de $26 \mathrm{~s}$ en ampoules). Par ailleurs, notre résultat présente une analogie avec l'observation de Finley et Fields (1962). Ces auteurs ont décrit une " dormance induite "sur les spores de FS 1518 par les températures sublétables inférieures à $100^{\circ} \mathrm{C}$ que nous avons retrouvée avec notre préparation de spores dans les mêmes conditions. Dans le cas du traitement dans notre appareil, il pourrait s'agir du même phénomène. La dormance serait induite par le séjour à $65^{\circ} \mathrm{C}$ pendant $2 \mathrm{mn}$ environ dans la section de pré-chauffage. Le court séjour à $111^{\circ} \mathrm{C}$ ne serait pas suffisant pour lever cette dormance, et même pourrait l'accentuer du fait qu'il constitue lui-même un traitement thermique sublétal.

De l'ensemble de ce travail, il ressort que le traitement thermique avec injection dans la vapeur a un effet inactivateur particulier, tout au moins entre 102 et $115^{\circ} \mathrm{C}$, plus fort que l'effet destructeur déduit des résultats des chauffages des spores en ampoules. En particulier, l'utilisation de chauffages dans des appareils U.H.T. à injection dans la vapeur à des températures inférieures à $120^{\circ} \mathrm{C}$ entraîne une forte inactivation des spores de $\mathrm{Cl}$. tyrobutyricum. Cette observation pourra peut-être être mise à profit pour la fabrication des fromages à partir des laits de vaches nourries avec des fourrages ensilés.

\section{Remerciements}

Nous remercions MM. Mocquot et Bergère pour les suggestions et critiques qu'ils ont faites à propos de ce travail. Nous remercions également MM. Grosclaude, Vermeire, Degas, Cavarroc et Lelubre qui ont imaginé et réalisé la vanne d'injection et réalisé les modifications de l'installation de stérilisation; MM. Lebars et Métro pour leur collaboration technique; et $M$. Tomassone et Mme Tassencourt du Laboratoire de Biométrie pour l'établissement du programme et la réalisation des calculs.

Reçu pour publication en décembre 1971.

\section{Références bibliographiques}

Aiba (S.), Humphrey (A. E) and Millis (N. F.) (1965). - Biochemical engineering. Academic Press, New-York, p. 209.

Burton (H.) (1958). - An analysis of the performance of an ultra-high-temperature milk sterilizing plant. II. Calculation of the bactericidal effectiveness. J. Dairy Res., 26, 324-337.

Burton (H.) (1970). - Comparison of milks processed by the direct and indirect methods of ultra-high-temperature sterilization. III. A note on the results for spore destruction obtained with an experimental ultra-high-temperature milk sterilizer. J. Dairy Res., 37, 227-231. 
Busta (F. F.) (1967). - Thermal inactivation characteristics of bacterial spores at ultra-high temperatures. Appl. Microbiol., 15, 640-645.

CERF (O.) (1969). - Les Clostridium des produits laitiers : problèmes posés par leur numération, mesure de leur thermorésistance. Thèse $3^{e}$ Cycle Nutrition, Caen.

Cerf (O.), Bergere (J. L.) et Hermier (J.) (1967). - Thermorésistance des spores de Clostridium tyrobutyricum et Clostridium butyricum. J. Dairy Res., 34, 221-230.

Edwards (J. L.) Jr., Busta (F. F.) and SPECK (M. L.) (1965). - Heat injury of Bacillus subtilis spores at ultra-high temperatures. Appl. Microbiol., 13, 858-864.

Esty (J. R.) and MeYeR (K. F.) (1922). - The heat resistance of the spores of Bacillus botulinus and allied anaerobes. XI. J. inf. Dis, 31, 650-665.

FinLEY (N.) and FieLDS (M. L.) (1962). - Heat activation and heat-induced dormancy of Bacillus stearothermophilus spores. Appl. Microbiol., 10, 231-236.

Franklin (J. G.), Underwood (H. M.), Perkin (A. G.) and Burton (H.) (1970). - Comparison of milks processed by the direct and indirect methods of hultra-hightemperature sterilization. II. The sporicidal efficiency of an experimental plant for direct and indirect processing. J. Dairy Res., 37, 219-226.

Hermier (J.) (1962). - La germination de la spore de Bacillus subtilis. I. Action des sucres et des acides aminés sur la phase initiale de la germination. Ann. Inst. Pasteur, Paris, 102, 629-643.

Hermier (J.), Verge (J.) et Grosclaude (G.) (1959). - Détermination de la durée de chauffage dans un appareil de stérilisation du lait à très haute température. Le Lait, 39, 20-32.

Hirsch (A.) and GRINSTED (E.) (1954). - Methods for the growth and enumeration of anaerobic spore formers from cheese, with observations on the effect of nisin. J. Dairy Res., 21, 101-110.

Miller (I.) und KandLER (O.) (1967). - Temperatur- und Zeit- Abhängigkeit der Sporenabtötung im Bereich der Ultrahocherhitzung. Milchwissenschaft, 22, 686-691.

Pflug (I. J.) and Schmidt (C. F.) (1968). - Thermal destruction of microorganisms, in LaWRenCe (C. A.) and Block (S. S.). Disinfection, sterilization and preservation, Lea and Febiger, Philadelphie, p. 63-105.

WANG (D. I. C.) (1963). - The kinetics of death of bacterial spores at elevated temperature. $P h$. D. Thesis, University of Pennsylvania.

Wilder (C. J.) and NoRdAN (H. C.) (1957). - A micro method and apparatus for the determination of rates of destruction of bacterial spores subjected to heat and bactericidal agents .Food Res., 22, 462-467. 